ANNALES

POLONICI MATHEMATICI

$85.3(2005)$

\title{
A new necessary condition for analytic varieties satisfying the local Phragmén-Lindelöf condition
}

\author{
by ToBias HeinRich (Düsseldorf)
}

\begin{abstract}
For an analytic variety $V$ in $\mathbb{C}^{n}$ containing the origin which satisfies the local Phragmén-Lindelöf condition $\mathrm{PL}_{\text {loc }}(0)$ it is shown that for each real simple curve $\gamma$ and each $d \geq 1$ the limit variety $T_{\gamma, d} V$ satisfies the strong Phragmén-Lindelöf condition (SPL).
\end{abstract}

1. Introduction. In their article [4] Braun, Meise, and Taylor derived necessary geometric conditions for an analytic variety in $\mathbb{C}^{n}$ to satisfy the local Phragmén-Lindelöf principle at its real points. They have shown that these conditions are sufficient for analytic curves in $\mathbb{C}^{2}$ and analytic surfaces in $\mathbb{C}^{3}$. For that purpose they develop the concept of limit varieties along a simple curve which are approximations of a certain order to the variety itself in conoids around the curve. In this context the question appears how properties of the variety pass over to limit varieties.

According to [4, Proposition 3.5], it is necessary for an analytic variety $V$ in $\mathbb{C}^{n}$ satisfying $\mathrm{PL}_{\text {loc }}(0)$ that for each real simple curve $\gamma$ and each $d \geq 1$ the limit variety $T_{\gamma, d} V$ satisfies $\mathrm{PL}_{\text {loc }}(\xi)$ at each of its real points $\xi$. A priori it is not clear whether $T_{\gamma, d} V$ has real points at all.

As we will see in Theorem 10 even more is true: If $V$ satisfies $\mathrm{PL}_{\text {loc }}(0)$, then for each real simple curve $\gamma$ and each $d \geq 1$ the limit variety $T_{\gamma, d} V$ has to satisfy the strong Phragmén-Lindelöf condition (SPL). According to Meise and Taylor [9, Proposition 4.4], this implies $\mathrm{PL}_{\mathrm{loc}}(\xi)$ at any real point $\xi \in T_{\gamma, d} V$. Moreover it follows from another result of Braun, Meise, and Taylor that $T_{\gamma, d} V$ has real points if it is not empty.

In Section 2 we recall the required geometric notions, while the main result is stated in Section 3. We conclude with an example.

The results of this article will be part of the author's doctoral thesis.

2000 Mathematics Subject Classification: 31C10, 32C25.

Key words and phrases: Phragmén-Lindelöf principle, extremal functions. 
2. Preliminaries. Throughout this paper $|\cdot|$ will denote the Euclidean norm on $\mathbb{C}^{n}$. For $a \in \mathbb{C}^{n}$ and $r>0$ let $B(a, r):=\left\{z \in \mathbb{C}^{n}:|z-a|<r\right\}$. For the geometric notions required below we refer to Chirka [7] and for example to Braun, Meise, and Taylor [4].

An analytic variety $V$ in $\mathbb{C}^{n}$ is defined to be an analytic subset of some open set in $\mathbb{C}^{n}$. Denote by $V_{\text {reg }}$ (resp. $V_{\text {sing }}$ ) the set of regular (resp. singular) points in $V$. If the dimension (see [7, 2.3 and 2.4]) at all points of $V$ equals $k \in \mathbb{N}$, then $V$ is said to be of pure dimension $k$.

Definition 1. A simple curve $\gamma$ in $\mathbb{C}^{n}$ is a map $\left.\gamma:\right] 0, \alpha\left[\rightarrow \mathbb{C}^{n}\right.$ which for some $\alpha>0$ and some $q \in \mathbb{N}$ admits a convergent Puiseux series expansion

$$
\gamma(t)=\sum_{j=q}^{\infty} \xi_{j} t^{j / q} \quad \text { with }\left|\xi_{q}\right|=1
$$

A real simple curve is a simple curve with range contained in $\mathbb{R}^{n}$.

Definition 2. Let $V$ be an analytic variety of pure dimension $k \geq 1$ in $\mathbb{C}^{n}$ which contains the origin, let $\left.\gamma:\right] 0, \alpha\left[\rightarrow \mathbb{C}^{n}\right.$ be a simple curve, and let $d \geq 1$. Then for $t \in] 0, \alpha[$ we define

$$
V_{\gamma, d, t}:=\left\{w \in \mathbb{C}^{n}: \gamma(t)+t^{d} w \in V\right\}=\frac{1}{t^{d}}(V-\gamma(t)),
$$

and we define the limit variety $T_{\gamma, d} V$ of $V$ along $\gamma$ as the set

$$
T_{\gamma, d} V:=\left\{\begin{array}{l|l}
\zeta \in \mathbb{C}^{n} & \begin{array}{l}
\zeta=\lim _{j \rightarrow \infty} z_{j}, \text { where } z_{j} \in V_{\gamma, t_{j}, d} \text { for } j \in \mathbb{N}, \\
\text { and } \left.\left(t_{j}\right)_{j \in \mathbb{N}} \text { is a null-sequence in }\right] 0, \alpha[
\end{array}
\end{array}\right\} .
$$

If it is clear from the context we will just write $V_{t}$ instead of $V_{\gamma, d, t}$.

Definition 3. Let $V$ be an analytic variety in $\mathbb{C}^{n}$ and let $\Omega$ be an open subset of $V$. A function $u: \Omega \rightarrow[-\infty, \infty$ [ is called plurisubharmonic if it is locally bounded above, plurisubharmonic in the usual sense on the regular points of $V$ in $\Omega$, and satisfies

$$
u(z)=\limsup _{\zeta \in \Omega_{\mathrm{reg}}, \zeta \rightarrow z} u(\zeta)
$$

at the singular points $z$ of $V$ in $\Omega$. We denote by $\operatorname{PSH}(\Omega)$ the set of all plurisubharmonic functions on $\Omega$.

Our aim is to relate the following two Phragmén-Lindelöf principles:

Definition 4. For $\xi \in \mathbb{R}^{n}$ and $r_{0}>0$ let $V \subset B\left(\xi, r_{0}\right)$ be an analytic variety in $\mathbb{C}^{n}$ which contains $\xi$. We say that $V$ satisfies the condition $\mathrm{PL}_{\text {loc }}(\xi)$ if there exist positive numbers $A$ and $r_{0} \geq r_{1} \geq r_{2}$ such that each $u \in$ $\operatorname{PSH}\left(V \cap B\left(\xi, r_{1}\right)\right)$ satisfying 
( $\alpha) u(z) \leq 1, z \in V \cap B\left(\xi, r_{1}\right)$,

( $\beta) u(z) \leq 0, z \in V \cap \mathbb{R}^{n} \cap B\left(\xi, r_{1}\right)$,

also satisfies

$(\gamma) u(z) \leq A|\operatorname{Im} z|, z \in V \cap B\left(\xi, r_{2}\right)$.

REMARK. In 1973 Hörmander [8] used the local Phragmén-Lindelöf principle to characterize the surjectivity of linear partial differential operators with constant coefficients on the space of real analytic functions on $\mathbb{R}^{n}$. The condition $\mathrm{PL}_{\text {loc }}$ is considered for example in Braun, Meise, and Taylor [4], where necessary conditions in any dimension which are sufficient in dimension two and three are derived.

Definition 5. Let $V$ be an algebraic variety in $\mathbb{C}^{n}$. We say that $V$ satisfies the condition (SPL) if there exists $A \geq 1$ such that each $u \in \operatorname{PSH}(V)$ satisfying

( $\alpha) u(z) \leq|z|+o(|z|), z \in V$,

(ß) $u(z) \leq 0, z \in V \cap \mathbb{R}^{n}$,

also satisfies

$(\gamma) u(z) \leq A|\operatorname{Im} z|, z \in V$.

REMARK. For equivalent characterizations of (SPL) we refer to Braun, Meise, and Taylor [1]. Its meaning for linear partial differential operators is explained in Meise and Taylor [9] and Braun, Meise, and Taylor [2].

\section{3. (SPL) on limit varieties as a necessary condition for a variety} to satisfy $\mathrm{PL}_{\mathrm{loc}}(0)$. Braun, Meise, and Taylor have shown in [3] that the condition (SPL) can be characterized by estimates for extremal functions which are defined as follows:

Definition 6. (a) For $D$ a domain in $\mathbb{C}^{n}, h$ a continuous plurisubharmonic function on $D, V$ an analytic variety in $\mathbb{C}^{n}$ which is contained in an open set containing $D$, and $E$ a subset of $D$, the extremal function of $E$ relative to $V, h, D$ is defined as

$$
U_{E}(z ; h, V, D):=\sup \left\{\begin{array}{l|l}
u(z) \mid \begin{array}{l}
u \in \operatorname{PSH}(V \cap D), \\
u \leq h \text { on } V \cap D \text { and } u \leq 0 \text { on } V \cap E
\end{array}
\end{array}\right\} .
$$

(b) For an analytic variety $V$ in $\mathbb{C}^{n}$ and $B, \varrho>0$ we set $U^{\varrho}(z, V, B):=\sup \left\{\begin{array}{l|l}\varphi(z) \mid \begin{array}{l}\varphi \in \operatorname{PSH}(V), \\ \varphi(w) \leq \min (|w|+B, \varrho|\operatorname{Im} w|) \text { for all } w \in V\end{array}\end{array}\right\}$.

We introduce some notations which will be used in the following: 
Notation 7. (a) For $R, \varepsilon, B>0$ define

$$
K(R, \varepsilon, B):=\left\{z \in \mathbb{C}^{n}:|\operatorname{Im} z| \leq \varepsilon(|z|+B),|z| \leq R / 2\right\} .
$$

(b) For $z \in \mathbb{C}^{n}$ we set $h(z):=|z|+B$.

We recall [10, Lemma 2.9]:

Lemma 8. The function $H: \mathbb{C}^{n} \rightarrow \mathbb{R}, H(z):=\frac{1}{2}\left(|\operatorname{Im} z|^{2}-|\operatorname{Re} z|^{2}\right)$, is plurisubharmonic and has the following properties:

(a) $H(z) \leq|\operatorname{Im} z|,|z| \leq 1$,

(c) $H(x) \leq 0, x \in \mathbb{R}^{n}$,

(b) $H(z) \leq|\operatorname{Im} z|-\frac{1}{2},|z|=1$,

(d) $H(i y) \geq 0, y \in \mathbb{R}^{n}$.

As a first step the next lemma provides estimates for local extremal functions.

Lemma 9. For $r_{0}>0$ let $V \subset B\left(0, r_{0}\right)$ be an analytic variety of pure dimension $k \geq 1$ in $\mathbb{C}^{n}$ which contains the origin. Suppose $V$ satisfies $\mathrm{PL}_{\text {loc }}(0)$ with positive numbers $A$ and $r_{0} \geq r_{1} \geq r_{2}$ as in Definition 4 . Let $\gamma:] 0, \alpha\left[\rightarrow \mathbb{R}^{n}\right.$ be a real simple curve and $d \geq 1$. Then for all $R, \varepsilon, B>0$ with $R \geq 2 B$ there exists $0<t_{0} \leq \alpha$ such that for each $0<t<t_{0}$,

$$
U_{K(R, \varepsilon, B)}\left(w ; h, V_{t}, B(0, R)\right) \leq 8 A|\operatorname{Im} w|, \quad w \in V_{t} \cap B(0, R / 8) .
$$

Proof. We may assume $0<r_{2} \leq r_{1}<1$. Then we can find $0<t_{0} \leq \alpha$ such that $t^{d} R / 8+r_{1} \leq 1$ and $\gamma(t)+t^{d}\left(V_{t} \cap B(0, R)\right) \subset V \cap B\left(0, r_{2}\right)$ for all $0<t<t_{0}$. Let $u \in \operatorname{PSH}\left(V_{t} \cap B(0, R)\right)$ satisfy $u \leq h$ on $V_{t} \cap B(0, R)$ and $u \leq 0$ on $V_{t} \cap K(R, \varepsilon, B)$. Note that any function appearing in the supremum on the left hand side of (1) has these properties. For $z \in \gamma(t)+t^{d}\left(V_{t} \cap B(0, R)\right)=$ $V \cap B\left(\gamma(t), t^{d} R\right)$ we define

$$
v(z)=v\left(\gamma(t)+t^{d} w\right):=u(w)=u\left(\frac{1}{t^{d}}(z-\gamma(t))\right) .
$$

If $z \in \gamma(t)+t^{d}\left(V_{t} \cap B(0, R / 2)\right)=V \cap B\left(\gamma(t), t^{d} R / 2\right)$ then $v(z)$ can be estimated as follows:

$$
v(z)=u(w) \leq|w|+B \leq \frac{R}{2}+\frac{R}{2}=R .
$$

Since $B(0, R / 2) \cap \mathbb{R}^{n} \subset K(R, \varepsilon, B)$ we see that for

$$
\begin{aligned}
& z \in \gamma(t)+t^{d}\left(V_{t} \cap \mathbb{R}^{n} \cap B(0, R / 2)\right) \\
& \quad=\mathbb{R}^{n} \cap\left[\gamma(t)+t^{d}\left(V_{t} \cap B(0, R / 2)\right)\right]=V \cap \mathbb{R}^{n} \cap B\left(\gamma(t), t^{d} R / 2\right)
\end{aligned}
$$

we have $v(z)=u(w) \leq 0$. 
Fix $w_{0} \in V_{t} \cap B(0, R / 8)$ and set $z_{0}:=\gamma(t)+t^{d} w_{0} \in V \cap B\left(\gamma(t), t^{d} R / 8\right)$. Let $r:=t^{d} R / 4$. Then each $z \in V$ with $\left|z-\operatorname{Re} z_{0}\right|<r$ satisfies

$$
\begin{aligned}
|z-\gamma(t)| & \leq\left|z-\operatorname{Re} z_{0}\right|+\left|\operatorname{Im} z_{0}\right|+\left|z_{0}-\gamma(t)\right| \\
& =\left|z-\operatorname{Re} z_{0}\right|+\left|\operatorname{Im}\left(z_{0}-\gamma(t)\right)\right|+\left|z_{0}-\gamma(t)\right| \\
& \leq\left|z-\operatorname{Re} z_{0}\right|+2\left|z_{0}-\gamma(t)\right| \\
& <r+2 t^{d} \frac{R}{8}=t^{d} \frac{R}{4}+t^{d} \frac{R}{4}=t^{d} \frac{R}{2},
\end{aligned}
$$

hence $z \in B\left(\gamma(t), t^{d} R / 2\right)$. Let $H$ be the function from Lemma 8. We define

$$
\widetilde{v}(z):=\max \left\{\frac{r}{2 R}\left(v(z)+2 R H\left(\frac{z-\operatorname{Re} z_{0}}{r}\right)\right),|\operatorname{Im} z|\right\}
$$

for $z \in V$ with $\left|z-\operatorname{Re} z_{0}\right|<r$, and $\widetilde{v}(z):=|\operatorname{Im} z|$ for $z \in V \cap B\left(0, r_{1}\right)$ with $\left|z-\operatorname{Re} z_{0}\right| \geq r$. For $z \in V$ with $\left|z-\operatorname{Re} z_{0}\right|=r$ we obtain from Lemma 8(b)

$$
\begin{aligned}
\frac{r}{2 R}(v(z)+2 R H( & \left.\left.\frac{z-\operatorname{Re} z_{0}}{r}\right)\right) \\
& \leq \frac{r}{2 R}\left(R+2 R\left(\left|\operatorname{Im} \frac{z-\operatorname{Re} z_{0}}{r}\right|-\frac{1}{2}\right)\right)=|\operatorname{Im} z|,
\end{aligned}
$$

hence $\widetilde{v} \in \operatorname{PSH}\left(V \cap B\left(0, r_{1}\right)\right)$. For $z \in V$ with $\left|z-\operatorname{Re} z_{0}\right|<r$ it follows from Lemma 8(a) that

$$
\begin{aligned}
\frac{r}{2 R}\left(v(z)+2 R H\left(\frac{z-\operatorname{Re} z_{0}}{r}\right)\right) & \leq \frac{r}{2 R}\left(R+2 R\left|\operatorname{Im} \frac{z-\operatorname{Re} z_{0}}{r}\right|\right) \\
& =\frac{r}{2}+|\operatorname{Im} z| \leq t^{d} \frac{R}{8}+r_{1} \leq 1
\end{aligned}
$$

for $t<t_{0}$. The hypothesis on $u$ and Lemma $8(\mathrm{c})$ imply that $\widetilde{v}(z) \leq 0$ on $V \cap \mathbb{R}^{n} \cap B\left(0, r_{1}\right)$, so $\widetilde{v}$ satisfies the conditions $(\alpha)$ and $(\beta)$ in the definition of $\mathrm{PL}_{\text {loc }}(0)$. Hence $\widetilde{v}(z) \leq A|\operatorname{Im} z|$ for $z \in V \cap B\left(0, r_{2}\right)$. According to Lemma $8(\mathrm{~d})$ we can write

which implies

$$
\frac{r}{2 R} u\left(w_{0}\right)=\frac{r}{2 R} v\left(z_{0}\right) \leq \widetilde{v}\left(z_{0}\right) \leq A\left|\operatorname{Im} z_{0}\right|
$$

$$
u\left(w_{0}\right) \leq \frac{2 R}{r} A\left|\operatorname{Im} z_{0}\right|=\frac{2 R}{t^{d} R / 4} A\left|\operatorname{Im}\left(\gamma(t)+t^{d} w_{0}\right)\right|=8 A\left|\operatorname{Im} w_{0}\right|,
$$

so estimate (1) holds for any $w_{0} \in V_{t} \cap B(0, R / 8)$.

The proof of the following theorem is based on the proof of Braun, Meise, and Taylor [3, Theorem 3.12].

TheOREM 10. For $r>0$ let $V \subset B(0, r)$ be an analytic variety of pure dimension $k \geq 1$ in $\mathbb{C}^{n}$ which contains the origin. If $V$ satisfies $\mathrm{PL}_{\text {loc }}(0)$, then $T_{\gamma, d} V$ satisfies (SPL) for each real simple curve $\gamma$ and each $d \geq 1$. 
Proof. According to [3, Lemma 3.2(b) and Lemma 3.5], $T_{\gamma, d} V$ has the property (SPL) if and only if there exists $A^{\prime} \geq 1$ such that for each $\varrho>0$ and each $B>0$ the following condition holds:

$$
U^{\varrho}\left(z, T_{\gamma, d} V, B\right) \leq A^{\prime}|\operatorname{Im} z|, \quad z \in\left(T_{\gamma, d} V\right)_{\text {reg }} .
$$

Fix $B, \varrho>0$. Let $u \in \operatorname{PSH}\left(T_{\gamma, d} V\right)$ satisfy $u(z) \leq \min (|z|+B, \varrho|\operatorname{Im} z|)$ for $z \in$ $T_{\gamma, d} V$. Let $\varepsilon>0$. If $z \in K(R, \varepsilon, B)$ then $\varrho|\operatorname{Im} z| \leq \varrho \varepsilon(|z|+B) \leq \varrho \varepsilon(B+R / 2)$, hence $u-\varrho \varepsilon(B+R / 2) \leq 0$ on $T_{\gamma, d} V \cap K(R, \varepsilon, B)$. For $h(z)=|z|+B$ and $w \in T_{\gamma, d} V \cap B(0, R)$ it follows that

$$
U^{\varrho}\left(w, T_{\gamma, d} V, B\right) \leq U_{K(R, \varepsilon, B)}\left(w ; h, T_{\gamma, d} V, B(0, R)\right)+\varrho \varepsilon(B+R / 2) .
$$

Fix $w \in\left(T_{\gamma, d} V\right)_{\text {reg }}$ and choose sequences $\left(t_{j}\right)_{j \in \mathbb{N}}$ in $\left[0, \infty\left[\right.\right.$ with $\lim _{j \rightarrow \infty} t_{j}=0$ and $\left(w_{j}\right)_{j \in \mathbb{N}}$ satisfying $w_{j} \in V_{t_{j}}$ for each $j \in \mathbb{N}$ and $\lim _{j \rightarrow \infty} w_{j}=w$. Choose $R>\max (8|w|, 2 B)$; then $(V \cap B(0, R))_{j \in \mathbb{N}}$ converges to $T_{\gamma, d} V \cap B(0, R)$ in the sense of [12, Definition 4.3], according to [4, Theorem 2.7(d)]. Note that the following estimates are valid for any $\varepsilon>0$. Hence [12, Theorem 4.4] (see also [3, Theorem 3.8]) implies

$$
U_{K(R, \varepsilon, B)}\left(w ; h, T_{\gamma, d} V, B(0, R)\right) \leq \liminf _{j \rightarrow \infty} U_{K(R, \varepsilon, B)}\left(w_{j} ; h, V_{t_{j}}, B(0, R)\right) .
$$

For $w \in B(0, R / 8)$ we may assume $w_{j} \in B(0, R / 8)$ for almost all $j \in \mathbb{N}$. Denote by $A$ the constant from Definition 4 . Then we can apply (1) to the right hand side of (4) and obtain

$$
U_{K(R, \varepsilon, B)}\left(w ; h, T_{\gamma, d} V, B(0, R)\right) \leq 8 A|\operatorname{Im} w| .
$$

Together with (3) this yields

$$
U^{\varrho}\left(w, T_{\gamma, d} V, B\right) \leq 8 A|\operatorname{Im} w|+\varrho \varepsilon(B+R / 2) .
$$

Since this holds for any $\varepsilon>0$, the claim is proved with $A^{\prime}=8 A$.

Remark 11. It follows from Theorem 10 and the proof of $[3$, Corollary 3.15] that for each variety $V$ satisfying $\mathrm{PL}_{\text {loc }}(0)$, each real simple curve $\gamma$, and each $d \geq 1$ for which $T_{\gamma, d} V$ is not empty, the intersection $T_{\gamma, d} V \cap \mathbb{R}^{n}$ is not empty.

Using arguments of Meise, Taylor, and Vogt (see [11, proof of Theorem 2.3]) one can show that Hörmander's characterization of the surjectivity of a linear partial differential operator with constant coefficients on all real analytic functions (see [8, following Theorem 1.3]) implies the local Phragmén-Lindelöf principle. Hence we obtain as a necessary condition:

Corollary 12. Let $P(D): \mathcal{A}\left(\mathbb{R}^{n}\right) \rightarrow \mathcal{A}\left(\mathbb{R}^{n}\right)$ be a linear partial differential operator with constant coefficients acting on the space $\mathcal{A}\left(\mathbb{R}^{n}\right)$ of all real analytic functions on $\mathbb{R}^{n}$. Assume that $P(D)$ is surjective. Then for the zero set $V\left(P_{m}\right)$ of the principal part of $P$ the following holds: For each $\xi \in V\left(P_{m}\right) \cap \mathbb{R}^{n}$ with $|\xi|=1$, each real simple curve $\gamma$ in $\mathbb{R}^{n}$, and each 
$d \geq 1$ the limit variety $T_{\gamma, d}\left(V\left(P_{m}\right)-\xi\right)$ satisfies (SPL), provided that it is not empty.

The following example shows that the result of Theorem 10 is an improvement of the one obtained by Braun, Meise, and Taylor in [4], where $\mathrm{PL}_{\text {loc }}(\xi)$ at any real point $\xi \in T_{\gamma, d} V$ is shown to be necessary (as one part of hyperbolicity in conoids) for $V$ to satisfy $\mathrm{PL}_{\mathrm{loc}}(0)$.

EXAmple 13. Define $f(x, y, z) \in \mathbb{C}[x, y, z]$ as

$$
f(x, y, z):=\left(y^{2}-x^{2}\right)\left(y^{2}-2 x^{2}\right)^{2}+x y^{2} z^{6}+z^{12}
$$

and let $V:=V(f)$. We claim that $V$ does not satisfy $\mathrm{PL}_{\text {loc }}(0)$. To show this, fix $\xi:=(0,0,1)$ and the real simple curve $\gamma(t):=t \xi, t>0$. We use [5, Proposition 4.3] to obtain the following limit varieties:

$$
\begin{aligned}
& T_{\gamma, d} V=T_{0} V=\left\{(x, y, z) \in \mathbb{C}^{3}:\left(y^{2}-x^{2}\right)\left(y^{2}-2 x^{2}\right)^{2}=0\right\}, \quad d \in[1,2[, \\
& T_{\gamma, 2} V=\left\{(x, y, z) \in \mathbb{C}^{3}: g(x, y):=y^{6}-5 x^{2} y^{4}+\left(8 x^{4}+x\right) y^{2}-4 x^{6}+1=0\right\}, \\
& T_{\gamma, d} V=\emptyset, \quad d>2 .
\end{aligned}
$$

We show that $T_{\gamma, 2} V$ does not satisfy (SPL); then it follows from Theorem 10 that $V$ cannot satisfy $\mathrm{PL}_{\mathrm{loc}}(0)$. Let $g_{m}$ denote the principal part of $g$. According to [6, Proposition 15], the zero set $V(g) \subset \mathbb{C}^{2}$ of $g$ satisfies (SPL) if and only if the following two conditions hold:

(a) $V(g)$ satisfies $\mathrm{PL}_{\text {loc }}(\xi)$ at each $\xi \in V(g) \cap \mathbb{R}^{2}$.

(b) For each $\eta, \zeta \in S^{1}$ satisfying $g_{m}(\zeta) \neq 0$ and $\operatorname{span}_{\mathbb{R}}(\eta, \zeta)=\mathbb{R}^{2}$ there exists $R>0$ such that for each $t \in \mathbb{R}$ with $|t| \geq R$, the polynomial $\lambda \mapsto g(t \eta+\lambda \xi)$ has only real zeros.

Note that $g(x, y)=h\left(x, y^{2}\right)$ for $h(x, y)=y^{3}-5 x^{2} y^{2}+\left(8 x^{4}+x\right) y-4 x^{6}+1$, and that for fixed $x \in \mathbb{R}$ each zero of $g(x, y)$ is real if and only if each zero of $h(x, y)$ is real and non-negative. Since $\operatorname{deg} h(x, \cdot)=3$ it suffices to calculate the discriminant to decide where $h(x, \cdot)$ has three real zeros. To normalize $h(x, \cdot)$ substitute $y=z+5 x^{2} / 3$ to obtain $\widetilde{h}(x, z)=z^{3}+3 p z+2 q$ with $3 p=-x^{4} / 3+x$ and $2 q=2 x^{6} / 27+5 x^{3} / 3+1$. Then the discriminant is defined as $D:=q^{2}+p^{3}=2 x^{9} / 27+25 x^{6} / 26+47 x^{3} / 54+1 / 4$. From $D>0$ for $x>0$ it follows that $h(x, \cdot)$ has only one real zero, which implies that $g(x, \cdot)$ has non-real zeros. Hence $V(g)$ fails (b), so $V(g)$ does not satisfy (SPL). This implies the claim for $T_{\gamma, 2} V=V(g) \times \mathbb{C}$.

However, we can show that condition (a) is satisfied: Each point of $T_{\gamma, 2} V$ is regular, since the gradient of $g$,

$$
\operatorname{grad} g(x, y)=\left(\begin{array}{c}
-10 x y^{4}+\left(32 x^{3}+1\right) y^{2}-24 x^{5} \\
2 y\left(3 y^{4}-10 x^{2} y^{2}+8 x^{4}+x\right)
\end{array}\right),
$$


has no zeros in common with $g$. This implies that $T_{\gamma, 2} V$ satisfies $\mathrm{PL}_{\text {loc }}(\xi)$ at any real point $\xi \in T_{\gamma, 2} V$.

\section{References}

[1] R. W. Braun, R. Meise, and B. A. Taylor, The algebraic surfaces on which the classical Phragmén-Lindelöf theorem holds, preprint.

[2] —, - - - Algebraic varieties on which the classical Phragmén-Lindelöf estimates hold for plurisubharmonic functions, Math. Z. 232 (1999), 103-135.

[3] - , - - Characterization of the linear partial differential equations that admit solution operators on Gevrey classes, J. Reine Angew. Math., to appear.

[4] - - - - - The geometry of analytic varieties satisfying the local Phragmén-Lindelöf condition and a geometric characterization of the partial differential operators that are surjective on $\mathcal{A}\left(\mathbb{R}^{4}\right)$, Trans. Amer. Math. Soc. 365 (2004), 1315-1383.

[5] - - - - , Higher order tangents to analytic varieties along curves, Canad. J. Math. 55 (2003), 64-90.

[6] - , - - - Optimal Gevrey classes for the existence of solution operators for linear partial differential operators in three variables, J. Math. Anal. Appl. 272 (2004), 852-868.

[7] E. M. Chirka, Complex Analytic Sets, Kluwer, 1989.

[8] L. Hörmander, On the existence of real analytic solutions of partial differential equations with constant coefficients, Invent. Math. 21 (1973), 151-183.

[9] R. Meise and B. A. Taylor, Phragmén-Lindelöf conditions for graph varieties, Results Math. 36 (1999), 121-148.

[10] R. Meise, B. A. Taylor, and D. Vogt, Continuous linear right inverses for partial differential operators on non-quasianalytic classes and on ultradistributions, Math. Nachr. 180 (1996), 213-242.

[11] - - - - Equivalence of analytic and plurisubharmonic Phragmén-Lindelöf conditions, in: Proc. Sympos. Pure Math. 52, Amer. Math. Soc., 1991, Part 3, 287-308.

[12] - , - - Extremal plurisubharmonic functions of linear growth on algebraic varieties, Math. Z. 219 (1995), 515-537.

Mathematisches Institut

Heinrich-Heine-Universität

Universitätsstrasse 1

40225 Düsseldorf, Germany

E-mail: heinrich@math.uni-duesseldorf.de 